\title{
PER UN RIESAME DELLA LEGAZIONE PAPALE IN SPAGNA NEL 1112
}

POR

\author{
Glauco Maria Cantarella \\ Universidad de Bolonia
}

\begin{abstract}
RESUMEN
En la Historia Compostellana se dice que en el año $1112 \mathrm{cl}$ abad de San Michele della Chiusa (dioc. Torino) llegó en legación a Galicia y a Santiago de Compostela. Algunos historiadores han sostenido que se trató, en realidad, del abad de Cluny, pero la edición crítica de la Historia (1988) excluye por completo esta interpretación. Recientes estudios han conseguido un mejor conocimiento de la historia de la abadía piamontesa y cs posible ahora reexaminar la cucstión.
\end{abstract}

\begin{abstract}
The Historia Compostellana inform us, that in 1112 the abbot of San Michele della Chiusa (dioc. Torino) went as papal Iegate to Galicia and to Santiago de Compostela. Various historians have conjectured that he was actually the abbot of Cluny; but following the critical edition of the Historia (1988), which cxcludes absolutely this interpretation, and in the wake of new studies that have cxpanded the history of this Piedmontesc abbey, it is now the time to reconsider the question.
\end{abstract}

1. «His igitur atque aliis Romanus Pontifex a Compostellane sedis episcopo multotiens suppliciter ammonitus uenerabilem personam, scilicet Clusensem abbatem, ad Hyspanos misit. Qui ab eodem legis magistro litteras secum attulit, quatinus omnes Hyspaniarum pontifices usque ad proximam beate Marie festiuitatem ad presentiam ipsius Pape conuenirent et Aragonensis regis et nobilis regine V. nuntios secum ducerent, ut, utriusque causa pleniter inda- 
gata, tantis cladibus, Deo auxiliante, finis imponeretur et Hispanie finibus pax et tranquillitas reformaretur»' ${ }^{\prime} E^{\prime}$ il 1112 . Il legato papale si avvia a compiere la sua missione e scopre che la situazione è molto più complicata di quanto sapesse: «Supradictus itaque Clusensis abbas occidentales partes ingrediens et multo plura et nefandiora quam, quod audierat, uidet totamque Hispaniam discordie stimulo perturbatam inuenit». Consegna, dunque, le lettere di cui è latore ${ }^{2}$ e infine rende visita a Diego Gelmírez, ${ }^{3}$ che fornisce all'inviato apostolico la propria versione: «His auditis, Clusensis abbas pontificis prudentiam nimium collaudauit acceptoque $a b$ eo consilio ab apostolica urbe discessit» ${ }^{4}$.

2. La Historia Compostellana è chiarissima: quel legato era l'abate di San Michele della Chiusa. La sua missione lo proiettò nel bel mezzo dei complicatissimi problemi che agitavano la Spagna settentrionale in quegli anni (il bellicoso matrimonio fra Urraca e Alfonso el Batallador, le ambizioni di Diego Gelmírez, vescovo di Compostella, sulla Galizia e sullo stesso re minorenne Alfonso VII, le ribellioni delle città). E' possibile, si domandò nel $1957 \mathrm{Ch}$. $\mathrm{J}$. Bishko, che un incarico di tanta delicatezza e importanza fosse affidato a «a minor Piedmontese abbot»? $?^{5}$ Che cosa avrebbe potuto capire di una situazione talmente intricata per la cui comprensione erano necessarie conoscenze vaste (oltre a capacità diplomatiche che non siamo in grado di giudicare, perché nessuno ce ne parla) ${ }^{6}$ Domanda legittima, cui lo studioso propose una risposta ipotetica e verosimile: si potrebbe ipotizzare che $\mathbf{i}$ copisti abbiano inteso male l'abbreviazione del cronista e che il dettato sia da leggere diversamente: non già «Clusensis» bensì Cluniacensis. Quel legato, allora, sarebbe Ponzio di Melgueil, abate di Cluny dal 1109 al 1122, che certamente, per le molteplici

1 Historia Compostellana, ed. E. FALQUE REY, Corpus Christianorum, Continuatio Mediaevaiis LXX, Turnhoult 1988, I.LXXIX.2, p. 12232-41.

2 Historia Compostellana, ed. cit., I.LXXIX.3, p. 12351-54, 72-76: «Preterea Clusensis abbas litteras a Romano Pontifice directas Hispaniarum episcopis tradidit et eos ad supradictam sinodum tempore determinato inuitauit, tu de regis et regine negotio et de pacis atque concordie colloquio in presentia ipsius Pape plenius tractarent».

3 Hist. Comp. 1.LXXIX.4, p. 12479-84: «abbas ad Gallitie partes proficiscitur et per angustas et asperas fauces montium Gallitiam ingreditur. Tandem ad apostolicam ciutatem Compostellam pernuenit et ab ejusdem loci pontifice honorifice susceptus est. Et qui Clusensis abbas uenerabilem ecclesie beati lacobi episcopum prudentie proflumm fontem habere nouerat...s.

4 Hist. Comp. I.LXXIX.5, p. $125137-139$.

s $\mathrm{CH}$. J. BISHKo, The Spanish Journey of Abbot Ponce of Cluny, «Ricerche di Storia Religiosa» I, 1957 (= Studi in onore di Giorgio la Piana), p. 314: «the case against accepting this teading of the text and recognizing a minor Piedmontese abbot, relatively unknown in western Spain, as Paschal II's agent for resolving a major political and military crisis, is very strong".

- Scarne notizie su Emmengaldo, abate di San Michele della Chiusa, in P.F. KeHR, Regessa Pontificun Romanorum. Italia Pontificia, VI,II, Berlino 1914, pp. 125-126: «a. 1112 a Paschale II in Hispaniam missus est» (p. 126).

La Personalidad Internacional de los Papas

Hispania Sacra 48 (1996) 
relazioni che i cluniacensi, insediati lungo la via di Compostella, avevano da decenni nella penisola iberica, avrebbe potuto dispore di conoscenze migliori e di informazioni più dirette sulla situazione (tanto più, potremmo aggiungere, se, come non è improbabile, Ponzio aveva conosciuto negli anni trascorsi nel monastero di. Saint-Pons-de-Thomières Ramiro, fratello di Alfonso d'Aragona, che vi si era monacato nel 1093$)^{7}$. Ma allora sarebbe più appropriato pensare al $1113^{8}$. Questa ipotesi appare affascinante, il che forse ha contribuito a far sí che non sia mai stata messa seriamente in discussione ${ }^{9}$ : nel 1978 essa è stata ripresa, seppure con qualche interrogativo, da H.E.J. Cowdrey ${ }^{10}$, e pochi anni fa è stata fatta propria, seguendo il Cowdrey, anche da chi scrive questa no$\mathrm{ta}^{11}$.

Ma lo studioso inglese aggiungeva: «It is wiser to suspend judgement in the absence of a critical edition' '? toria Compostellana non dovrebbe più lasciare spazio a quell'interpretazione. Il testo e le sue varianti sono chiarissimi! in quei brani si parla proprio dell'abate Clusensis e non di quello Cluniacensis. Sarebbe ben curioso che sulla forma di questa parola, che ricorre almeno una cinquantina di volte nella $\mathrm{His}$ toria $^{13}$, si equivocasse solo a proposito di quell'episodio. Dobbiamo ammettere dunque che è stato Ermengaldo di San Michele della Chiusa (dioc. Torino) e non Ponzio di Cluny a svolgere quella legazione in Spagna.

3. La domanda del Bishko si ripropone intera: perché «a minor Piedmontese abbot»? Senonché recenti studi ci offrono la possibjlità di ripensare alle

7 Cfr. H. Salvador Martínez, La rebelión de los burgos. Crisis de Estado y coyuniura so)cial, Madrid 1992, p. 116; Ponzio era stato monaco a Saint-Pons: cfr. H.E.J CowDREY, $T_{w o}$ Siudies in Cluniac History 1049-1/26, «Studi Gregoriani» XI (1978), p. 194. - Non siamo ritusciti a vedere R.A. Fletcher, Saint James' Catapul. The Life and Times of Diego Gelmirez of Santiago de Compostela, Oxford 1984.

* BiSHxo, The Spanish Journey cit., p. 314ss. («Thuss Abbot Ponce was the natural, not to say the inevitable, papal choice for the task of restoring peace to the troubled Spain»: p. 315).

9 Qualche dubbio era stato espresso, a nostra conoscenza, solo da C. Servatius, Pascialis $/ /$. (1099-J/18). Studien zus seiner Person und seiner Politik, Stuttgart 1979, p. 127.

10) Cfi. Cowdrey, Two Siudies in Chiniac History cit., pp. 200-201, 274.

"G.M. CANTARELLA, I monaci di Climy, Torino 1993, pp. 239-240. Non solo: anche recensendo il volume del SALVADOR MARTíNEZ e riprovando che l'A. non avesse fatto menzione dell'potesi Bishko-Cowdrey (cfr. «Studi Medievali» $3^{\mathrm{a}}$ s., XXXIV [1993], p. 275). Eppure parecchi anni fa avevamo scritto di quell'ipotesi; «non... sembra accettabile, benché indubbiamente suggestiva» (G.M. CANTARElla, Pietro il Venerabile, Cluny, i monasteri cluniacensi dell'falia settentrionale: un altro aspetto della crisi del monachesimo nel XII secolo?, in Cluny in Lombarlia, I, Cesena 1979, p. 41I n. 79); quando si dice della capacità di fascinazione e di trascinamento meccanico di certe jdee "facili"!

12 Cowdrey, Two Studies in Cluniac History cit, p. 201, cont. n. 76.

is Salvo etrore: cff. Hist. Comp., Index Locorum, s.v. Claniacum, Clusensis abbas. 
dimensioni di quell'abbazia sulle Alpi e alle sue relazioni e ci mostrano una realtà molto più complessa di quanto non fosse dato di sapere quarant'anni or sono.

San Michele, fondata da Ugo Dissutus di Montboissier nel 983-987 su un importante itinerario di valico e di pellegrinaggio, era stata collegata con il monachesimo cluniacense sin dagli inizi: il suo primo abate proveniva dal monastero Lézat, riformato da Odone di Cluny ${ }^{14}$. Con ciò, naturalmente, essa non entrò a far parte della ecclesia clumiacensis (che del resto in quei decenni era appena in formazione): ma i rapporti fra le due abbazie non dovettero venir meno e comunque furono ripresi nella seconda metì dell'XI secolo e tenuti ben vivi nel corso del XII, come testimonia una significativa serie di indizi.

Verso il 1123 Rinaldo di Vézelay compose la sua Vita di Ugo di Semur (Ugo I di Cluny, 1049-1109); è l'ultima, forse, di una serie iniziata per volontà di Ponzio intorno al 1120 , ma coincide con un periodo di grande crisi istituzionale e sembra piuttosto voler ricordare a quali altezze fosse giunta la ecclesia cluniacensis grazie all'opera del santo ${ }^{15}$ : un'esortazione, insomma, a non perdere di vista il proprio passato e anzi a trarne ispirazione per il futuro. Come gli agiografi che l'avevano preceduto Rinaldo non ricorda molte delle straordinarie circostanze storiche alle quali Ugo era stato presente (ad esempio la sua partecipazione alla penitenza di Canossa nel 1077), ma, nel tracciare una sorta di Uberblick del sistema di grandi abbazie che erano state aggregate a Cluny, aggiunse che egli aveva salvato con il suo santo consiglio l'abbazia di San Michele della Chiusa, desolata dalle tempeste e funestata dal fulmine. Sarà soltanto una coincidenza che proprio quell'abbazia, e non un'altra qualsiasi, venisse ricordata in quel contesto che sembra scritto a memoria e vademecum per i cluniacensi smarriti dopo le forzate dimissioni di Ponzio e la rapida successione di Ugo Il e di Pietro di Montboissier? Ma concediamo pure che si sia trattato di una coincidenza (reiterata, però, nella Synopsis metrica di quella $V i t a)^{16}$. Nel 1162 l'abate di San Michele, Stefano di Boulogne, che era

if G. SERGt, Laristocrazia della preghiera. Politica e scehe religiose nel medieve italiano, Roma 1994, pp. 107, 75, 90.

is Cfr. CowDrey, Two Studies in Cluniac History cit., pp. 22-29; cfi. il nostro Chmy tra passato e futuro nelle «Vite di sant Ugo, in Cluny e il suo abate Ugo. Splendore e crisi di an grande ordine monastico, a cura di G.M. CANTARELLA-D. TUNIZ, Milano-Novara 1982, pp. 19-20. Non va inserita a questa "serie" la Vita del cosiddelto Anonimo II, composta in Inghilterra verso la metà del XII secolo e che a nostro avviso va inscritta nella polemica con i cisterciensi (ci permettiamo di rinvinte ancora una volta ad un nostro lavoro: II monachesimo it Oecidente: il pieno Medioevo [secoli XI-XII], in La Storia. I grandi problemi dal Medisevo all'Età Contemporanea I.I, Torino 1988, p. 351).

is Dobbiamo rinviare ancora ai nostri Pietro il Venerabile, Chmy cit, pp. 410-411; Culhra ed ecclesiologia a Cluny (sec. XII), «Aevum» LV (1981), p. 286 e n. 109 .

La Personalidad Internacional de los Papas

Hispania Sacra 48 (1996) 
stato «monachus Cluniacensis», venne chiamato come abate proprio a Cluny: una Cluny ancora una volta lacerata dalle divisioni interne e gravemente indebolita dallerrata scelta di campo di Ugo III di Fraisans nello scisma fra Alessandro III e Vittore IV. Nel 1172, poi, l'abate piemontese Benedetto III si fece promotore di una «specialis societas» con l'omologa abbazia normanna di Mont Saint-Michel, con Vézelay e con Cluny ${ }^{17}$. Si trattava di una forma di «affratellamento» (Verbriderung) tra monasteri che non implicava affatto la loro comune appartenenza ad una medesima struttura ma registrava un rapporto speciale tra di essi ${ }^{18}$ : un'operazione, in questo caso, resa particolarmente audace ed ambiziosa dal fatto che appena dieci anni prima Alessandro III aveva punito Cluny per la scelta di Ugo III e soddisfatto le vecchie aspirazioni dei monaci di Vézelay restituendo a quest'ultima la sua indipendenza ${ }^{19}$; l'abate chiusino dunque riconnetteva (nella forma impegnativa, ma non organizzativa, della societas) due abbazie che si erano appena separate, con l'evjdente proposito di ricomporre la concordia fraterna fra $\mathrm{i}$ monaci ristabilendo $\mathrm{i}$ rapporti ed impostandoli su di un piano diverso dall'ancora recente passato. Ma certamente non avrebbe nemmen potuto immaginare un progetto del genere se non avesse potuto contare sul riconoscimento di una posizione tutt'altro che «minore» nel mondo monastico francese.

Allora, forse, la reiterata menzione di San Michele nell'agiografia di Ugo I è meno incidentale di quanto volessimo supporre. Le relazioni con Cluny, evidentemente strettissime nella seconda metà del XII secolo, dovevano essere avvertite come particolari e forti anche nei decenni precedenti. L'abbazia della Chiusa doveva essere collegata da un rapporto abbastanza speciale con la grande Cluny dell'età di Ugo I e di Ponzio di Melgueil. Nell'età di Pietro il Venerabile (abate di Cluny tra il 1122 e il 1156 ) poi potrebbero essersi ancora consolidate, visto che tra i suoi antenati c'era proprio il fondatore del monastero piemontese 20 .

4. Ci sembra che questo renda all'abbazia di San Michele della Chiusa ed al suo abate Ermengaldo una dimensione molto più appropriata. E dato che entia non sunt multiplicanda praeter necessitatem, come insegnò Guglielmo d'Ockham, tentiamo di rivedere la questione con i dati che le appartengono, cercando di ragionare sulle implicazioni che essi possono suggerire.

$17 \mathrm{Cfr}$. SerGi, Laristocrazia della preghiera cit., pp. $89-90$.

I8 Cfi: ancora SERGI, Liaristocrazia della preghiera, cle parla di: «tendenza lederativa monaslica sovraregionale» (p. 90$)$.

15) JL 10720 ( 1162 maggio 17, Montpellier).

20 G. Constable, The Letters of Peter the Venerable, 11, Cambridge, Mass., 1967, pp. 234-235. 
Innanzitutto va ristabilita la datazione: che, non essendo più in causa Ponzio di Melgueil, dev'essere restituita al 1112. E poi: un prelato come quello piemontese, in relazioni con Cluny senza essere cluniacense, avrebbe potuto forse garantire una rappresentanza, sebbene non ufficiale, proprio a quegli interessi cluniacensi che si trovavano coinvolti nella bufera (basterà pensare alle vicende di Sahagún del $1110-1112)^{21}$ senza trovarsi direttamente implicato in questioni che lo riguardassero direttamente. Ermengaldo di San Michele della Chiusa come procuratore di Cluny? Forse non è necessario arrivare a tanto. Certo egli sembra un personaggio sul crinale di molte esigenze: non appartenendo alla Curia romana né a Cluny poteva apparire come uomo non pregiudizialmente schierato al riguardo della complessa situazione con la quale sarebbe venuto a contatto, ma essendo in relazioni con ambedue poteva essere ugualmente informato, e altrettanto utile perché avrebbe potuto fornire preziose informazioni di prima mano. Non dimentichiamo, inoltre, che non sappiamo nulla sull'uomo, sulla sua provenienza e sulle sue relazioni familiari e personali.

E, soprattutto, restituiamo alla Historia Compostellana il suo senso, non sopravvalutando quanto il cronista scrive e quindi senza perdere di vista il fatto che è molto preciso nel delimitare i compiti di questo legato, che fa apparire non già come un plenipotenziario (come era stato, ad esempio, nel 1090 il cardinale Raniero di Bleda, che nel 1112 regnava con il nome di Pasquale II $)^{22}$ ma piuttosto come uno strumento per far giungere a destinazione le lettere papali: uno strumento sicuro perché autorevolmente costituito in legazione ufficiale. Tutt'altra cosa dal «for resolving a major political and military crisis» del Bishko!

Il problema rimane aperto: ma, quanto alla presenza di Ponzio di Cluny in Galizia nel 1113, dobbiamo riconoscere che non se ne può più parlare.

Per interpretare quella vicenda sarà necessario fare ricorso ad altre categorie e ad altre motivazioni; ad esempio si potrebbe pensare ad una precisa intenzione di Pasquale II, la cui personale esperienza avrebbe potuto suggerirgli l'inutilità di inviare dei legati con pieni poteri in una situazione tanto complessa: la decisione ultima, in fin dei conti, sarebbe stata presa a Roma (così comera avvenuto a lui stesso, con Urbano II). E per convocare le parti neIl'Urbe non era necessario molto di più che un messaggero ufficiale e gerarchicamente autorevole non tanto per la sua posizione nella Chiesa quanto per l'incarico di cui era investito, di fronte al quale $i$ destinatari non potessero

21 Cfr. Salvador MaRtínez, La rebelión de los burgos cit., pp. 225-261.

22 Sulla legazione spagnola di Raniero cfr. SERvatius, Paschalis //. (/099-///8) cit., pp. 26-32, 119-120. Abbiamo ripteso la questione anche nel nostro Pasquale II e il suo tempo, in corso di stampa presso l'ed. Ligtori (Napoli, 1996).

La Personalidad Internacional de los Papas Hispania Secra 48 (1996) 
negarsi di ricevere l'ingiunzione papale. Non c'era proprio bisogno, per questo, di un ecclesiastico particolarmente in vista, bensì di un uomo capace di prendere $\mathrm{i}$ contatti secondo le istruzioni ricevute, di vedere e riferire. $\mathrm{E}$ che non avesse una propria politica in Spagna, suscettibile di influenzare i suoi giudizi e la relazione che avrebbe reso alla Sede Apostolica. 